\title{
Ultrafast spectroscopy of graphene-protected thin copper films
}

\author{
Eva A. A. Pogna ${ }^{1}$, Stefano Dal Conte ${ }^{1}$, Giancarlo Soavi ${ }^{1,2}$, \\ Vasyl G. Kravets ${ }^{3}$, Yong-Jin Kim ${ }^{3}$, Stefano Longhi ${ }^{1,4}$, \\ Alexander N. Grigorenko ${ }^{3}$, Giulio Cerullo ${ }^{1,4}$, Giuseppe Della Valle ${ }^{1,4 *}$ \\ ${ }^{1}$ Dipartimento di Fisica, Politecnico di Milano, Piazza L. da Vinci 32, 20133 Milano, Italy \\ ${ }^{2}$ Cambridge Graphene Centre, Engineering Department University of Cambridge, 9, JJ \\ Thomson Avenue Cambridge, CB3 OFA, UK \\ ${ }^{3}$ School of Physics and Astronomy, University of Manchester, Manchester, M13 9PL, UK \\ ${ }^{4}$ Istituto di Fotonica e Nanotecnologie, Consiglio Nazionale delle Ricerche, Piazza L. da \\ Vinci 32, 20133 Milano, Italy \\ E-mail: giuseppe.dellavalle@polimi.it
}

\begin{abstract}
We study by broad-band pump-probe spectroscopy the ultrafast optical response of thin copper films covered by a monolayer of graphene. It is demonstrated that graphene protection does not alter the thermo-modulational nonlinearity of copper in the whole visible range. Also, we provide a quantitative validation of a theoretical model for this optical nonlinearity, derived from a semiclassical description of electron thermalization dynamics and subsequent modulation of copper dielectric function from $450 \mathrm{~nm}$ to 700
\end{abstract}

\footnotetext{
${ }^{*}$ To whom correspondence should be addressed
} 
$\mathrm{nm}$ wavelength. Our results extend to the nonlinear domain the capability of grapheneprotected copper nanolayers to serve as a low cost optical grade material, with major potential impact in nonlinear plasmonics and metamaterials.

\section{Keywords}

Ultrafast Spectroscopy, Metal Optics, Graphene, Nonlinear Optics, Hot Electrons

With the rapid development of the novel research fields of plasmonics and metamaterials, metals have witnessed a true renaissance. The unique features of plasmonic resonances in metal-based nanostructures have disclosed unprecedented opportunities in photonics, from nanofocusing to deep sub-wavelength guiding of light ${ }^{1,2}$, with ubiquitous applications in nanoscience and nanotechnology (see the overviews given in Refs. 3-9). Also, metallic optical structures of different kind, including thin metallic films ${ }^{10,11}$, metal-dielectric multilayers $^{12}$, plasmonic metasurfaces ${ }^{13,14}$, plasmonic waveguides ${ }^{15}$, hybrid plasmonic-photonic crystal cavities ${ }^{16}$, and even single plasmonic nanoantennas ${ }^{17,18}$ have demonstrated the potential of providing ultrafast all-optical modulation of light, thanks to the exploitation of the giant optical nonlinearity in the metallic medium. It is worth noting that even though well known to exist since a long time (see the review paper of Ref. 19 and references therein), the giant optical nonlinearity of metals has been overlooked for many decades simply because of the lack of highly transparent metallic structures, enabled only recently by nanofabrication. These results are now paving the way to the development of a novel approach to nonlinear optics that is metal-based, with potential impact on a new generation of nonlinear metamaterials and plasmonic/nanophotonic devices (see the review paper of Ref. 20). Interestingly, despite of the availability of many metals, most of (and maybe the best) results reported in the field of linear and nonlinear metal optics deal with gold, which has become the optical metal. The reason for this preference is that metals are subject to oxidation, a process that compromises their optical quality, but gold is a noteworthy exception. However, the high production cost and the poor compatibility of gold with silicon-based technologies because of 
a very efficient diffusion of gold into silicon (see the review of Ref. 21 and references therein) are now acting as a bottleneck for further developments in the field, and many attempts have been reported to identify alternative metallic materials (see e.g. the review papers of Refs. 22-25). Heavily-doped semiconductors and graphene represent promising routes, at least for applications in the infrared, but for operation in the visible the stability and ease of nanostructuring offered by thin gold films is still by far superior.

Most recently, a new route has been proposed, that is the exploitation of more conventional existing metals where corrosion is prevented by mechanical protection. Grapheneprotection of thin films of strongly interacting metals like copper ${ }^{26,27}$, nickel, cobalt ${ }^{28}$, and silver ${ }^{27,29}$ has been demonstrated to preserve the optical quality of the metal surface against oxidation (see also Ref. 30 for an overview). The results reported for copper are of particular interest because the effectiveness of graphene protection has been demonstrated even in water environment, as ascertained by the excitation of high quality surface-plasmon-polariton (SPP) resonances ${ }^{27}$. Moreover, the quality factor of SPP resonances in graphene-protected copper turned out to be even better than in gold thin films, because of the better surface quality of copper films, now preserved by graphene protection. Compatibility of graphene protection of copper with the dielectric-loading technique for the manufacturing of plasmonic waveguides has been also demonstrated ${ }^{27}$. These results indicate that, because of its similarity to gold (almost same plasma frequency and interband optical transitions) copper can potentially replace gold in many plasmonic structures and metamaterials thanks to graphene-protection, with major benefits in terms of lower production costs and better compatibility with silicon based technologies.

Even though well assessed within the linear regime of the metallic optical response, suitability of graphene-protection of copper for nonlinear metal optics is still waiting for a validation. Actually, aside from other high figures of merit, graphene has demonstrated also a giant nonlinear optical response (see e.g. Refs. 31-33) that in graphene-metal composites can, in principle, interfere with the metal nonlinearity, and eventually compromise its 
exploitation. Therefore the capability of graphene-protected copper thin films to effectively exploit the giant optical nonlinearity of copper is an open issue of major relevance for the assessment of this new route to novel metallic media in optics (and eventual disposal of gold in favor of more convenient alternatives).

In this paper we address this issue by a comprehensive ultrafast spectroscopy study, in reflection and in transmission, of both graphene-protected and fresh pristine thin copper films. Our results indicate that graphene-protection does not alter the nonlinear properties of copper in the whole visible range. Also, we provide an experimental validation of the theoretical model for the thermo-modulational nonlinearity of copper in analogy with the one reported recently for gold ${ }^{11,34}$. We believe that our results can boost the exploitation of graphene-protection of copper and other common metals in metal-based nonlinear optical devices including ultrafast plasmonic and metamaterial nanostructures of the kind reported in Ref. 20.

\section{Experimental results}

Pristine copper thin films were deposited by electron-beam evaporation on top of a fusedsilica substrate. A 1.5-nm chromium layer was grown before copper evaporation to guarantee adhesion of the metal. A sketch of the sample cross-section is detailed in Fig. 1(a), whereas Fig. 1(b) reports the imaginary (top panel) and real (bottom panel) part of copper dielectric function measured on a typical sample with copper film thickness in the range of 40-50 nm. Within few days, the copper film degrades, forming inhomogeneous copper oxide that not only reduces the metal thickness but also compromises its optical quality ${ }^{35-37}$ [Fig. 1(c)]. To prevent oxidation, the freshly-made copper film was protected by growing $\mathrm{cm}$-size monolayer (ML) graphene by Chemical Vapour Deposition (see Methods for details). When observed in reflection, the protected film looks almost identical to a freshly-made unprotected copper film of the same thickness because of the poor absorption of the ML graphene $(\sim 3 \%)$ against 
the relatively high reflectivity of copper $(>50 \%)$ in the visible. This qualitative indication is confirmed by comparing the transmission spectrum of a protected sample with that of an unprotected one [cf. black circles and red triangles in Fig. 1(d)], the two samples having the same nominal value for the film thickness $d=45 \mathrm{~nm}$. In both samples the transmittance is dominated by a pronounced peak at around $580 \mathrm{~nm}$.

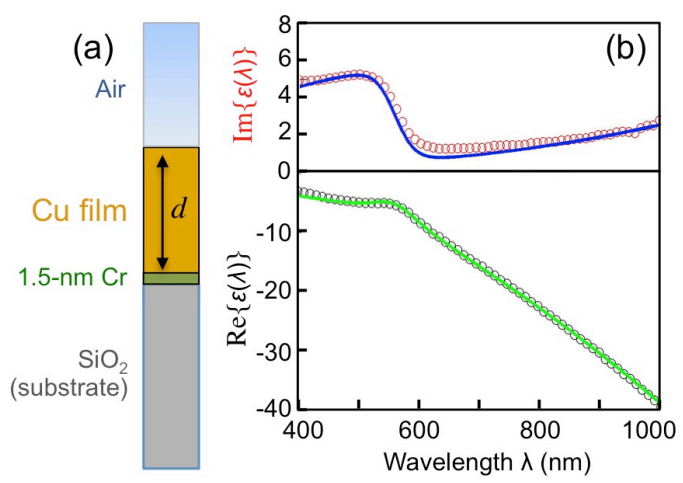

(c)
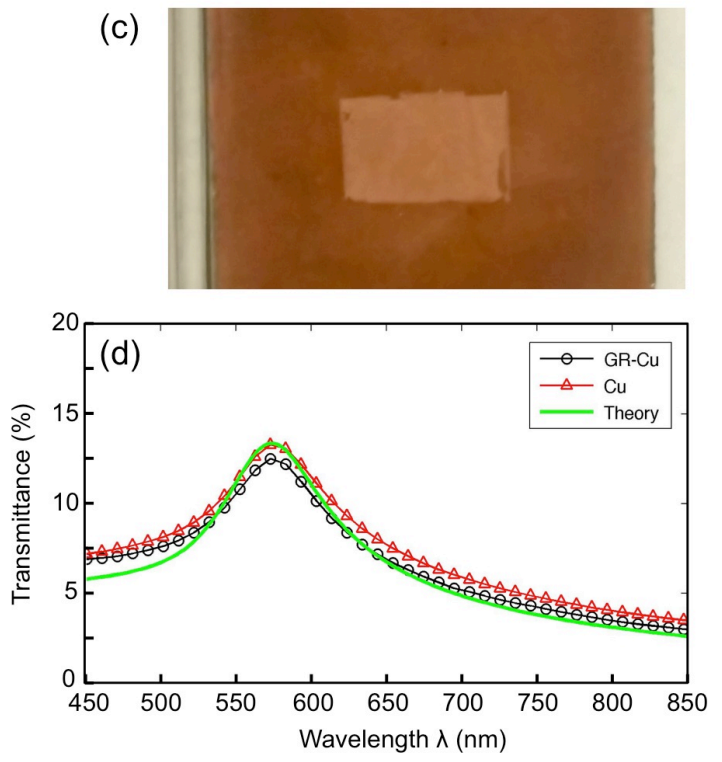

Figure 1: (a) Cross-section sketch of the pristine copper film sample. (b) Typical dielectric function of copper in a film of few tens nm thickness: experimental data from Ref. 27 (circles) are compared with numerical simulations (solid lines) after Eqs. (1)-(2). (c) An optical image of a graphene protected copper sample (a lighter rectangular in the middle) on a copper deposited slide after a month of fabrication. Notice visible deterioration of the non-protected copper (darker area around the rectangular) which is caused by oxidation. (d) Measured transmittance spectra of the pristine (red triangles) and graphene-protected (black circles) copper films in the whole visible range are compared with numerical simulations (green line). 

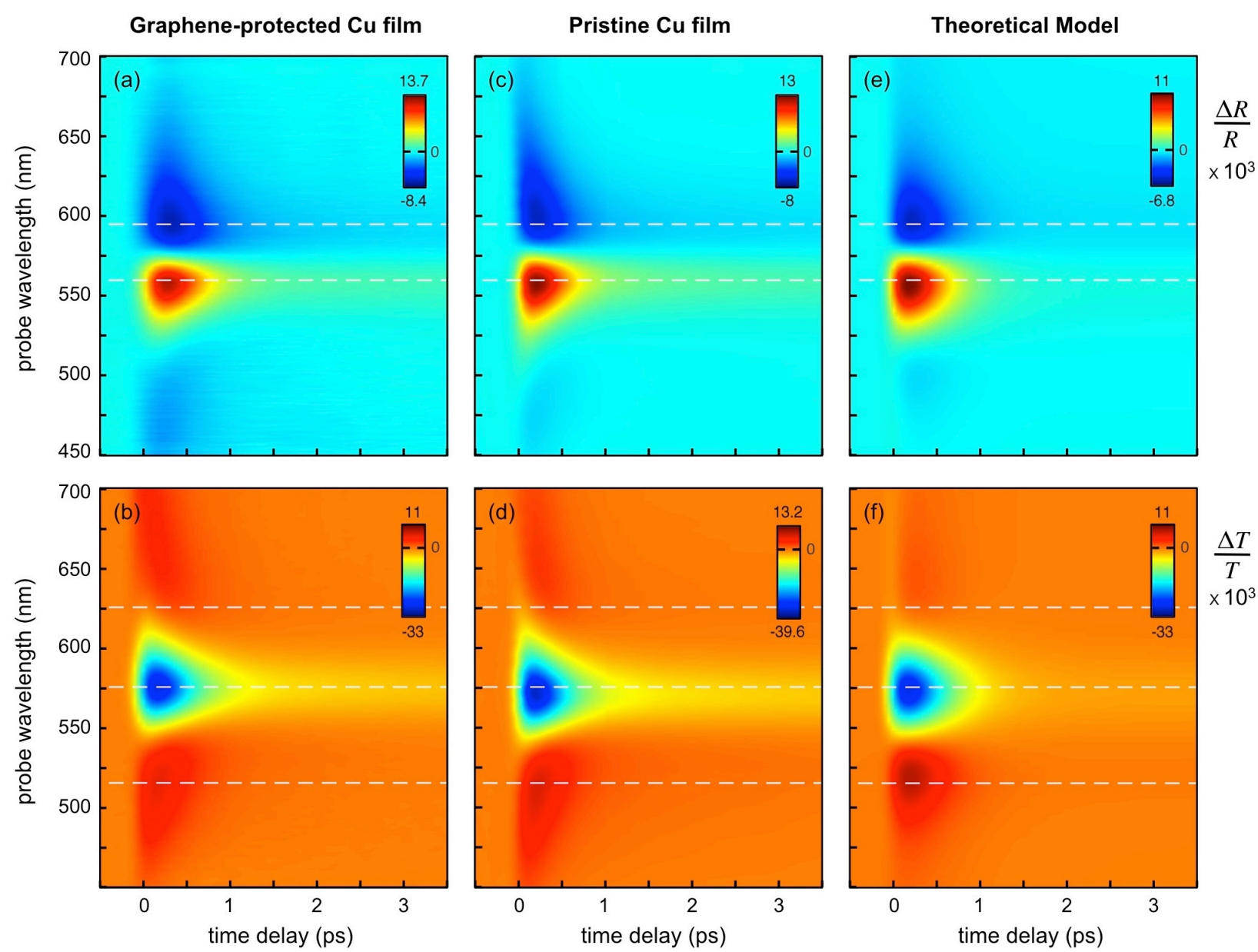

Figure 2: Time-resolved differential reflection (top panels) and transmission (bottom panels) measurements of the graphene-protected (left) and pristine (center) copper film in the visible. Right panels show corresponding numerical simulations performed on the pristine copper film. Dashed white lines indicate the cross-sections reported in Fig. 3.

To assess the suitability of graphene-protected copper not only for linear optical experiments but also in the nonlinear regime, we performed ultra-fast pump-probe spectroscopy on both the protected and the unprotected samples. The experimental setup is described in detail in the Methods. Briefly, we photoexcite the sample with a 100-fs infrared pump pulse centered at $800 \mathrm{~nm}$, and we monitor its reflectance and transmittance over the visible range by means of a white light supercontinuum probe, as a function of the time delay between the two pulses. Figure 2 shows the experimental differential reflection $(\Delta R / R)$ and differential transmission $(\Delta T / T)$ maps of the protected (left panels) and unprotected (center panels) copper films, under $\sim 1.2 \mathrm{~mJ} / \mathrm{cm}^{2}$ pump fluence. The temporal dynamics of the $\Delta R / R$ 
and of the $\Delta T / T$ at some selected wavelengths in the protected and unprotected films are detailed in Fig. 3(a)-(b) and Fig. 3(c)-(d), respectively. The measurements performed on the two samples both in reflection and in transmission are fully consistent, thus demonstrating that graphene protection does not alter the nonlinear optical response of the copper film in the whole visible range.

The time-frequency maps are rich of many distinctive features that can be summarized as follows: i) the $\Delta R / R$ signal is dominated by a positive lobe, peaked at around $560 \mathrm{~nm}$, surrounded by two minor negative lobes, the most prominent being peaked at around 595 $\mathrm{nm}$; ii) the $\Delta T / T$ signal is almost complementary to the $\Delta R / R$ signal, even though the dominating (negative) peak is red-shifted to $575 \mathrm{~nm}$; iii) the $\Delta R / R$ and $\Delta T / T$ signals share the same dynamics, with a build-up of the signal peak at around $250 \mathrm{fs}$ time delay and the decay to a long-lived plateau (almost stationary on the time scale of our experiment) within about $2-3$ ps.

The origin of above features can be identified in analogy with what reported in thin gold films (see e.g. Ref. 10). In particular, very recent pump-probe experiments on thin copper films confirm the idea that the thermo-modulational mechanism that dominates gold optical nonlinearity is also responsible for the transient optical response of copper films ${ }^{38}$. Absorption of the infrared pump pulse excites an out-of-equilibrium electron distribution in the conduction band. A rapid thermalization mainly driven by electron-electron interactions gives rise to hot electrons (described by a Fermi-Dirac distribution, but at a higher temperature as compared to the equilibrium one) that further thermalize with the metal lattice through electron-phonon interactions within few ps. The dynamics of energy exchange between hot electrons and the lattice is usually captured by the so-called two-temperature model (2TM), consisting of two coupled rate equations for the electrons and lattice temperatures $^{10,39}$. The excess energy accumulated in the lattice is then released to the environment on a much longer time-scale (of few hundred ps) by phonon-phonon scattering. The smearing of the electron distribution caused by pump absorption results into a modulation of the 


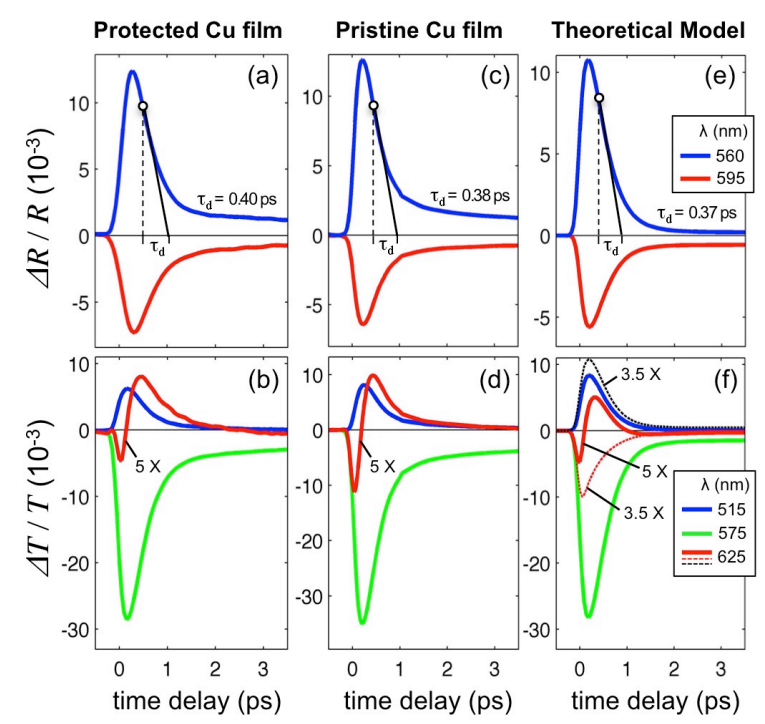

Figure 3: Cross-sections of the maps of Fig. 2 at some selected wavelengths (indicated by dashed white lines in the panels of Fig. 2). Panels (a), (c) and (e) also show the time constant $\tau_{d}$ of the signal decay at $\lambda=560 \mathrm{~nm}$, as estimated by fitting the $\Delta R / R$ trace with an exponential function (plus constant), starting from 150 fs after the signal peak (marked with an empty circle on the blu curves). The dynamics at $\lambda=625 \mathrm{~nm}$ in panel (b), (d) and (f) is 5 times magnified for better visualization. Dotted lines in panel (f) show the contributions to the $\Delta T / T$ at $625 \mathrm{~nm}$ arising from thermalized carriers (black) and non thermalized ones (red), 3.5 times magnified for better visualization.

interband transition rate, and in particular it increases (decreases) interband absorption below (above) the absorption edge, which for copper sits at around $2.17 \mathrm{eV}(\sim 570 \mathrm{~nm})^{40,41}$. The modulation of absorption (and subsequent modulation of the refractive index) induces a variation of the transmissivity $(\Delta T / T)$ and reflectivity $(\Delta R / R)$, as measured by the probe pulse (see Fig. 2).

In spite of this qualitative understanding, a detailed quantitative modeling of the transient optical response of thin copper films in the whole visible spectral range has been so far overlooked, contrary to gold films and nanostructures, that have been extensively investigated both in the visible and near infrared (see e.g. Refs. 10,11,17,18,42). Of course, the design of copper-based nonlinear optical structures demands a predictive model of the thermo-modulational nonlinearity of copper to allow engineering of the geometrical parameters. However, without graphene protection, a pristine copper film rapidly degrades by oxidation (even at room temperature), forming few $\mathrm{nm}$ thick $\mathrm{CuO}_{2}$ islands at the metal 
surface within few days ${ }^{35,36}$. This prevents any practical exploitation of the nonlinear modeling for design, which explains the poor attention so far deserved to this topic. With the advent of graphene-protection and subsequent long-term stability of copper, the interest for a detailed quantitative model of its thermo-modulational nonlinearity is renewed, and it is our aim to provide such a model in the following section.

\section{Theoretical model}

First of all, we introduce a model for the linear optical properties of copper films that will serve as a building block for the development of a transient (i.e. nonlinear) optical model. The complex dielectric function of copper (as that of gold and silver) in the visible and infrared can be modeled as the sum of two terms, i.e. $\epsilon=\epsilon_{D S}+\epsilon_{I B}$, with $\epsilon_{D S}$ the DrudeSommerfeld (DS) contribution belonging to free electrons in the conduction band, and $\epsilon_{I B}$ the contribution belonging to bound electrons via inter-band (IB) optical transitions ${ }^{43,44}$.

The (relative) DS permittivity as a function of the optical pulsation $\omega=2 \pi c / \lambda$ (with $\lambda$ the optical wavelength and $c$ the speed of light in vacuum) is given by:

$$
\epsilon_{D S}(\omega)=1-\frac{\omega_{P}^{2}}{\omega^{2}+\Gamma^{2}}+i \frac{\omega_{P}^{2} \Gamma}{\omega\left(\omega^{2}+\Gamma^{2}\right)}
$$

where $\omega_{P}$ and $\Gamma$ are two characteristic constants of the metal, referred to as the plasma frequency and Drude damping, respectively. We assumed $\omega_{P}=1.255 \times 10^{16} \mathrm{rad} / \mathrm{s}$ for copper, which is the average between the values reported by Ordal $^{45}$ and Zeman $^{46}$ (differing by less than 10\%). Contrary to $\omega_{P}$, an estimation of the Drude damping $\Gamma$ is quite critical if one considers the spreading of values reported in the literature. This uncertainty is due to the fact that many processes contribute to the Drude damping, including electron scattering to the grain boundaries in the polycrystalline phase of the metal, which in turns depends on the specific fabrication process (see e.g. Refs. 47,48 for the case of gold structures). Therefore, we assumed $\Gamma$ as a fitting parameter in the range of values reported in the literature, i.e. 
$5-15 \times 10^{13} \mathrm{rad} / \mathrm{s}^{45,46}$.

It is widely assumed that the IB contribution to the dielectric function of copper is dominated by optical transitions near the $L$-point in the Brillouin zone ${ }^{40,41}$, but, similarly to the case of gold ${ }^{49}$, we found that a contribution from optical transitions near the $X$-point ought to be included to retrieve good agreement with experimental data. By following a semiclassical approach (based on Fermi golden rule ${ }^{49,50}$ ) the imaginary part of the (relative) IB permittivity is computed as:

$$
\epsilon_{I B}^{\prime \prime}(\omega)=\frac{\pi e^{2}}{3 \epsilon_{0} m_{0}^{2}}\left[\frac{P_{L}^{2}}{\omega^{2}} J_{L}(\omega)+\frac{P_{X}^{2}}{\omega^{2}} J_{X}(\omega)\right] .
$$

In above equation, $J_{L}$ and $J_{X}$ are the joint density of states (JDOS) of the IB transition near $\mathrm{L}$ and $\mathrm{X}$ points the Brillouin zone, and $P_{L}$ and $P_{X}$ are the absolute values of the corresponding dipole matrix-elements (assumed to be constant in the considered energy range), whereas $\epsilon_{0}$ is the vacuum permittivity and $m_{0}$ the free electron mass. The JDOS functions are computed under parabolic approximation of the two bands with effective masses fitted on the ab-initio relativistic calculations of copper band-structure reported in Ref. 51. To take into account broadening effects in the IB transitions (mostly due to Drude scattering in the conduction states), similarly to the case of gold ${ }^{49}$, a smearing of the Fermi-Dirac function is introduced in the JDOS computation, by replacing the room temperature with an effective temperature $T_{0}^{*}=600 \mathrm{~K}$. Once the imaginary part $\epsilon_{I B}^{\prime \prime}$ is computed from Eq. (2), the real part $\epsilon_{I B}^{\prime}$ is retrieved by Kramers-Kronig transformation. Note that because of the constant matrix element approximation underlying Eq. (2) and the need for a broad range of electron energies to be considered in order to span all visible wavelengths, $P_{L}$ and $P_{X}$ ought to be fitted on the experimental data. When taking as experimental data the dielectric function reported in Ref. 27 , the fitting procedure retrieves: $\Gamma=10.7 \times 10^{13} \mathrm{rad} / \mathrm{s}, P_{L}=0.49 P_{L}^{A u}$ and $P_{X}=0.65 P_{X}^{A u}$, being $P_{L(X)}^{A u}$ the dipole matrix element of gold around $L(X)$ point $^{50}$. Note that the model well reproduces the measured dielectric function of copper in the whole 
visible range [cf. circles and solid line Fig. 1(b)]. The transmission of the pristine copper film sample is then numerically simulated by implementing the structure sketched in Fig.1 (a) with standard transfer-matrix method (TMM) of multi-layer media ${ }^{52}$. The numerically computed transmittance spectrum is shown as solid line in Fig. 1(d) and well compares with the measured spectra of the unprotected and protected samples.

This model for the linear dielectric function can be exploited to quantitatively describe the transient processes underlying the nonlinear optical response, according to a scenario that has been widely adopted for gold and silver, but so far overlooked for copper. The basic idea is that metal optical nonlinearity stems from an optically-induced thermo-modulation of the DS and IB dielectric functions.

Regarding the former, the absorption of an intense light pulse (the pump pulse in our experiment) results into heating of the metal lattice and thus into a variation of the Drude damping parameter according to the linear law $\Delta \Gamma\left(T_{l}\right)=\Gamma \beta\left(T_{l}-T_{0}\right)$, with $T_{l}$ the actual lattice temperature, $T_{0}$ the equilibrium temperature, and $\beta$ a characteristic parameter that can be estimated from the Debye temperature of the metal according to the Holstein model ${ }^{53}$. Then, according to Eq. (1), the $\Delta \Gamma$ translates into a variation $\Delta \epsilon_{D S}$ of the DS dielectric function.

The origin of a nonlinear mechanism dealing with the IB dielectric function can be understood according to the following argument. The JDOS function depends on the occupation probability $f(E)$ of states in the conduction band $(\mathrm{CB})$, according to the equation ${ }^{54}$ : $J(\omega)=\int_{C B} D(E, \omega)[1-f(E)] d E$, with $D(E, \omega)$ the energy distribution of the joint density of states (EDJDOS) for the considered IB transition. Any variation $\Delta f$ with respect to the equilibrium Fermi-Dirac distribution $f_{0}$ induced by light absorption (from the pump) translates into a $\Delta J$, which in turns results into a modification $\Delta \epsilon_{I B}^{\prime \prime}$ of IB dielectric function, according to Eq. (2), and corresponding $\Delta \epsilon_{I B}^{\prime}$ by Kramers-Kronig transformation.

In our pump-probe measurements, the total $\Delta \epsilon=\Delta \epsilon_{D S}+\Delta \epsilon_{I B}$ induced by the pump is then probed, at a certain time-delay $t$, by the weak probe pulse. Therefore, to quanti- 
tatively model the above described mechanisms of the optical nonlinearity, the key point is to compute the temporal dynamics of the lattice temperature, $T_{l}(t)$, and of the electrons occupation probability $\Delta f(E, t)$. A standard approach to determine the $\Delta f$ is based on numerical solution of Boltzmann equation ${ }^{10,17}$. Here we apply a simpler method that has been recently validated for gold ${ }^{11,12}$, allowing one to avoid numerical integration of Boltzmann equation, yet grasping the essentials of Boltzmann formalism in the relaxation time approximation. Within this model, the out-of-equilibrium electrons are split in two populations: hot thermalized electrons, described by a Fermi-Dirac occupation probability at a temperature $T_{e}$ (higher than the equilibrium temperature) and non-thermalized electrons. The latter are directly excited by pump-absorption and couples to thermalized electrons by electron-electron scattering and to the lattice by electron-phonon scattering. This way, it is possible to describe the electron and lattice heat dynamics by an extended version of the $2 \mathrm{TM}(\mathrm{E} 2 \mathrm{TM})$, that consists in two coupled equations for $T_{e}$ and $T_{l}$ to be solved numerically in the time domain (see Methods for details). From the subsequent $\Delta \epsilon_{I B}(\omega, t)$ and $\Delta \epsilon_{D S}(\omega, t)$, the dynamics of the $\Delta R / R$ and $\Delta T / T$ spectra of the pristine copper film configuration of Fig. 1(a) are simulated by standard TMM in the whole visible. The resulting maps are reported in Fig. 2(e)-(f) whereas Fig. 3(e)-(f) show some dynamics computed at specific wavelengths.

\section{Discussion and Conclusion}

Comparison between Fig. 2(c) and (e) and between Fig. 2(d) and (f) indicates an excellent agreement of our model with the experimental data in the whole visible range, both in reflection and in transmission. We found that the contribution to the transient optical

response arising from $\mathrm{X}$-point $\mathrm{IB}$ transition is negligible as compared to that belonging to the L-point, similarly to the case of gold ${ }^{49}$. Also, note that the temporal dynamics of Fig. 3(c) and (d) well compare with the theoretical ones, reported in Fig. 3(e) and (f), 
even on the sup-ps time scale, which is a remarkable achievement of the model, considering the simplification introduced in the electrons dynamics, as compared with the Boltzmann equation modeling. This suggests that copper transient optical response is less affected by non-thermalized carriers as compared to the case of gold, but to conclude on this point a dedicated study at higher temporal resolution is mandatory. However, it is worth noting that the contribution from non-thermalized carriers, even though weak, can be revealed in our measurements by inspecting the very initial dynamics in the red spectrum of the $\Delta T / T$ map. A magnified cross-section at $625 \mathrm{~nm}$ wavelength [red line in Figs. 3(b) and (d)] shows a nonmonotonic build-up of the signal, with an abrupt sign change within about 300 fs that can't be explained by a classic 2TM but is well captured by our E2TM, as detailed by Fig. 3(f). By disentangling the different contributions to the numerically computed $\Delta T / T$, it is found that non thermalized carriers give rise to a rapid negative transient [dotted red line in Fig. 3(f)] whereas thermalized carriers are responsible to a slower positive build-up [dotted black line in Fig. 3(f)]. In the considered wavelength range (i.e. far from IB absorption edge) the two contributions are comparable in magnitude and thus their superposition results into the characteristic sign change above detailed, similarly to what reported in thin gold films ${ }^{10,11}$ and nanostructures ${ }^{17,18}$.

The contribution arising from the modulation of the DS permittivity turns out to be much weaker than the one belonging to modulation of the IB transition, in the considered range of wavelengths. This is in agreement with what reported for gold thin films in the visible, confirming that also for copper the lattice dynamics could become relevant in the near-infrared (i.e. far from the IB transitions) or for very long time delays (after the vanishing of contributions from hot-electrons).

Note that our modeling of the thermo-modulational nonlinearity of copper thin films does not employ any fitting parameter (in addition to the few parameters used to fit the linear model).

One can argue whether the role of graphene as a passive protective layer for copper 
can be extended to the infrared or not, when dealing with nonlinear optical phenomena. Preliminary results are against this scenario. Fig. 4 shows the $\Delta R / R$ transient spectrum of the graphene-protected sample in the 840-960 nm wavelength range under $1.1 \mathrm{~mJ} / \mathrm{cm}^{2}$ pump fluence (panel a), compared to the $\Delta R / R$ from the pristine copper film under same excitation conditions (panel b). Note that the transient optical spectrum of the protected sample, with a spectrally broad positive ultrafast dynamics decaying on the sub-ps timescale [Fig. 4(a)], is by no means related to copper, which exhibits a negligible response (basically, not detectable at the pump fluence of the experiment) [Fig. 4(b)]. A cross-section of the $\Delta R / R$ map from the protected sample at $900 \mathrm{~nm}$ wavelength is reported in Fig. 4(c) (circles). A bi-exponential fit of the signal decay [red line in Fig. 4(c)] indicates that the dynamics is dominated by a fast process with time constant $\tau_{1} \sim 170 \mathrm{fs}$, superposed to a minor contribution from a slower process with time constant $\tau_{2} \geq 1$ ps. The origin of such features observed in the infrared is thus related to an active role of graphene in the ultrafast transient optical response of the protected sample.

The ultrafast optical response of ML graphene has been intensively studied (see e.g. Refs. 31-33) and is understood as follows: according to the band structure of graphene, absorption of a pump photon of frequency $h \nu_{p}$ promotes an electron from an energy $-h \nu_{p} / 2$ in the valence band to an energy $h \nu_{p} / 2$ in the conduction band. Ultrafast photoexcitation thus creates a strongly non-equilibrium (non-thermal) distribution of electrons in the conduction band (and holes in the valence band), consisting of two peaks at $\pm h \nu_{p} / 2$ with respect to the Dirac point. The primary relaxation process is thermalization due to electron-electron interaction, happening on the 10 -fs timescale, giving rise to an equilibrated Fermi-Dirac electron distribution with a well defined temperature, higher than that of the lattice. This time-evolving hot-electron distribution inhibits, due to Pauli blocking, the absorption of the probe pulse, thus yielding an increase in transmission (photobleaching). The hot electrons subsequently reach thermal equilibrium with the colder lattice via electron-phonon scattering. This process is known to feature the bi-exponential decay observed in our measurements, with a first 


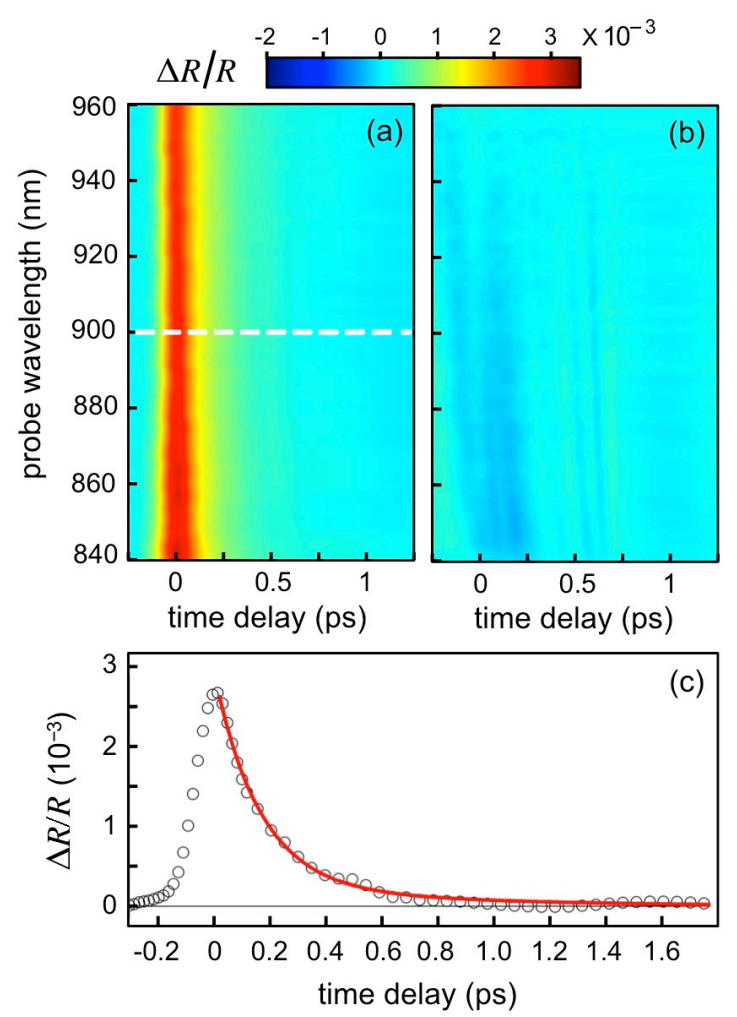

Figure 4: (a) Time-resolved differential reflection of (a) the graphene-protected copper film and (b) the pristine copper film in the near infrared. Panel (c) shows the cross-section of panel (a) at $900 \mathrm{~nm}$ wavelength (circles), and superposed bi-exponential fit of the decay dynamics (red curve).

time constant of about 160 fs (i.e. very close to the $\tau_{1}$ of our fit), assigned to the interaction with optical phonons, and a second longer time constant $\sim 1-2$ ps (in agreement with the estimated $\tau_{2}$ as detailed above) due to relaxation of the thermalized electron/phonon bath by anharmonic decay of the hot phonons. Since our temporal resolution is insufficient to monitor the initial electron-electron relaxation process, the measured transient optical response is dominated by the dynamics of Fermi-Dirac distribution of thermalized electrons, which peaks in the infrared and decays exponentially.

However, note that the pump-probe of monolayer graphene ${ }^{55}$ or thin graphite films ${ }^{56}$ deposited on a dielectric substrate retrieves a positive signal in transmission and a negative signal in reflection, for the present case of probe beam impinging from the air and not from the substrate (see also Ref. 57 for a detailed study on this issue). The data of Fig. 4(a) 
exhibits a counter-intuitive reversal of sign, meaning that the role of copper beyond the visible is thus still crucial in our graphene-protected film. Even though a disentanglement of the contributions arising from copper and from graphene in the infrared would deserve a dedicated modeling that is beyond the scope of the present paper, the sign reversal in the spectrum of Fig. 4(a) can be interpreted as follows. In light of the negligible transient optical response of copper shown in Fig. 4(b), the copper film basically operates as a linear material with very high reflectivity (exceeding $90 \%$ in this wavelength range according to TMM calculations). Therefore, the differential reflection experiment in the infrared actually probes a double-pass in the graphene ML caused by signal reflection at the copper surface. This explains why the $\Delta R / R$ of Fig. 4(a) well compares with typical $\Delta T / T$ spectra measured on pristine graphene samples ${ }^{55,56}$.

To conclude, we provided an extensive investigation of the transient optical response of graphene-protected thin copper films in the whole visible, both in reflection and in transmission. Comparison with the transient optical response of a pristine copper film ascertains that graphene protection (employed to prevent copper oxidation) preserves the giant optical nonlinearity of the metal from $450 \mathrm{~nm}$ to $700 \mathrm{~nm}$. We also achieved, for the first time to our knowledge, a quantitative validation of a predictive model for the thermo-modulational optical nonlinearity of copper. Contrary to the visible range, where the nonlinear optical response of graphene is weak (and thus the transient reflectivity of the graphene protected copper is dominated by the metal), in the near infrared (i.e. far from the IB transition of copper) the nonlinear response of the metal significantly decreases and that of graphene increases. This results into a much more complex scenario for the optical nonlinearity in the infrared, where the transient optical response of the composite material is dominated by graphene. It is envisaged that, at least in the visible, our results can be exploited for further developments in the field of nonlinear optics with metals, for example by replacing gold with graphene-protected copper in metal-dielectric nanostructures of the kind reported in Refs. $13,14,16$ for all-optical modulation and switching of light beams. Also, provided that the 
E2TM is reduced to an effective three temperature model similarly to the case of gold ${ }^{10}$, the procedure reported in Ref. 34 to retrieve an analytical expression for the third-order nonlinear susceptibility $\left(\chi^{(3)}\right)$ of gold could be straightforwardly extended to copper as well. With an espression for the $\chi^{(3)}$ at hand, one can exploit well assessed computational approaches of nonlinear optics (like, e.g., the Green-function formalism or the generalized nonlinear Schrödinger equation) to study nonlinear phenomena in copper-based plasmonic waveguides, in analogy with what recently reported for gold-based plasmonic waveguides ${ }^{50,58,59}$.

\section{Acknowledgments}

Financial support from the Fondazione Cariplo is acknowlegded by G.D.V. and S.L. through the project New Frontiers in Plasmonic Nanosensing (Grant No. 2011-0338). G.D.V. acknowledges COST Action MP1302-NanoSpectroscopy. G.C. and A.N.G. acknowledges support by the EC under Graphene Flagship (contract no. CNECT-ICT-604391).

\section{Methods}

\section{Sample fabrication.}

First, copper films with thickness of $45 \mathrm{~nm}$ were produced by electron-beam evaporation at a base pressure of about $10^{-5}$ mTorr and a growth rate of $0.3 \mathrm{~nm} / \mathrm{s}$ (film thickness was monitored by calibrated quartz microbalance). An electron-beam target of $99.99 \% \mathrm{Cu}$ from Sigma-Aldrich was used. A thin adhesion layer of $\mathrm{Cr}$ with thickness of about 1.5 nm was evaporated onto a substrate before copper. The glass substrates of sizes $25 \times$ $25 \mathrm{~mm}^{2}$ and thickness of $1 \mathrm{~mm}$ were used for all the studied samples. The substrates were ultrasonically cleaned in heated acetone and isopropanol before depositions. Second, large size graphene films were grown on $\mathrm{Cu}$ foil $(50 \mu \mathrm{m})$ by using the chemical vapour deposition (CVD) method $^{60}$. $\mathrm{Cu}$ foil was placed inside a quartz tube and then heated up to $1000^{\circ} \mathrm{C}$ with a $\mathrm{H}_{2}$ flow at rate of $20 \mathrm{~cm}^{3} / \mathrm{min}$ and a pressure of $200 \mathrm{mTorr}$. To remove the native 
oxide layer from surface, the $\mathrm{Cu}$ foil was first annealed at $1000^{\circ} \mathrm{C}$ during 30 minutes. Then a gas mixture of $\mathrm{H}_{2}$ and $\mathrm{CH}_{4}$, with flow rates of 20 and $40 \mathrm{~cm}^{3} / \mathrm{min}$, respectively, was introduced into the chamber. CVD growth was performed at a pressure of 600 mTorr during 30 mins. Finally, the CVD chamber was rapidly cooled to the room temperature in hydrogen atmosphere. Third, a large-area CVD graphene sheet was transferred onto the Cu film using wet-etching and transfer process ${ }^{27}$. A graphene sheet on top of $\mathrm{Cu}$ foil was covered by poly(methyl methacrylate) (PMMA) using spin-coating method, and then graphene with PMMA layers was separated from origin substrate by wet-etching of $\mathrm{Cu}$ foil. After this the CVD graphene films covered with supporting polymer layer was ready to transfer onto the $\mathrm{Cu}$ coated substrates. The PMMA layer was removed by acetone and the graphene surface was further cleaned by nitrogen blowing. Details of graphene transfer process are described elsewhere $^{27,60}$.

\section{Ultrafast optical spectroscopy.}

The setup starts from an amplified Ti:sapphire laser (Coherent, Libra 2), delivering 100-fs, $4-\mathrm{mJ}$ pulses at $800 \mathrm{~nm}$ and $2-\mathrm{kHz}$ repetition rate ${ }^{61}$. The laser fundamental wavelength provides the pump pulse which is modulated with a chopper at $1 \mathrm{kHz}$ and focalised on the sample with a spot of $250 \mu \mathrm{m}$ average radius. The probe pulse, instead, is a white-light continuum (spanning the 450-1000 $\mathrm{nm}$ range) generated in a sapphire plate. The sample is mounted onto a roto-translational stage allowing measurements both in reflection and in transmission by setting an angle of incidence of few degrees. An optical multichannel analyzer provides broadband detection of the reflected and transmitted probe pulse at the full $2-\mathrm{kHz}$ laser repetition rate. The time delayed is tuned by varying the optical path of the pump pulse with a motorized translation stage and the temporal resolution of the apparatus is of the order of $100 \mathrm{fs}$. The measured signal is a two-dimensional map of the relative differential reflectance $(\Delta R / R)$ or transmittance $(\Delta T / T)$ as a function of the probe wavelength and the pump-probe delay. More precisely, if $R_{o n}\left(T_{o n}\right)$ and $R_{o f f}\left(T_{o f f}\right)$ are the probe spectra reflected (transmitted) through the excited and the unexcited sample, respectively, the mea- 
sured signals are $\Delta R / R=R_{o n}(\lambda, t) / R_{\text {off }}(\lambda)-1$ and $\Delta T / T=T_{o n}(\lambda, t) / T_{\text {off }}(\lambda)-1$. In the experiments the pump and the probe pulse are linearly parallel polarized and impinge on the sample from the air side [see the sample sketch in Fig. 1(a)].

\section{Numerical modeling.}

The E2TM reads as follows ${ }^{62}$ :

$$
\begin{aligned}
\gamma T_{e} \frac{d T_{e}}{d t} & =-g\left(T_{e}-T_{l}\right)+\int P_{a}\left(t^{\prime}\right) H_{e e}\left(t-t^{\prime}\right) d t^{\prime} \\
C_{l} \frac{d T_{l}}{d t} & =g\left(T_{e}-T_{l}\right)+\int P_{a}\left(t^{\prime}\right) H_{e p}\left(t-t^{\prime}\right) d t^{\prime}
\end{aligned}
$$

In Eq. (3) $\gamma$ is the electrons heat capacity constant, $C_{l}$ is the lattice heat capacity, $g$ is the electron-phonon coupling constant, $P_{a}(t)$ is the pump pulse power absorbed in the unit volume of the metal and $H_{e e}(t)$ and $H_{e p}(t)$ account for the energy transfer from non-thermalized electrons (generated by pump excitation) to thermalized electrons and to the lattice, respectively (and whose explicit expression can be found in Refs. 11,62).

After numerical solution of Eq. (3), the variation of thermalized electrons temperature $\Delta T_{e}(t)=T_{e}(t)-T_{0}$ is used to compute the corresponding variation of occupation probability as $\Delta f_{T}(E, t)=f_{0}\left[E, T_{0}^{*}+\Delta T_{e}(t)\right]-f_{0}\left(E, T_{0}^{*}\right)$. For the non-thermalized carriers, the E2TM model provides ${ }^{62}$ :

$$
\Delta f_{N T}(E, t)=\delta_{N T}(E) \int_{-\infty}^{t} P_{a}\left(t^{\prime}\right) e^{-\left(t-t^{\prime}\right) / \tau(E)} d t^{\prime}
$$

where $\delta_{N T}(E)$ is a characteristic step-like spectral function for the intraband absorption process in the metal (see Refs. 11,62 for details) and $\tau(E)=\tau_{e p} \tau_{e e}(E) /\left[\tau_{e p}+\tau_{e e}(E)\right]$, with $\tau_{e e}(E)=\tau_{0} E_{F}^{2} /\left(E-E_{F}\right)^{2}$ and $\tau_{e p}=\tau_{f} h \nu_{p} /\left(k_{B} \theta_{D}\right)$. In above formulas, $h \nu_{p}=1.55 \mathrm{eV}$ is the photon energy of the pump in our experiments, whereas all the other parameters are defined in Table 1, with values retrieved by available literature on copper (see references in Table 1), except for $\tau_{0}$ for which we took the value in gold (since, to the best of our knowledge, it has 
never been estimated in copper). The absorbed pump power density $P_{a}(t)$ is computed from TMM calculation of the absorption in the thin copper film and a homogeneous thermal load in the film is assumed (see Ref. 12 on gold thin films for further details). From the computed $\Delta f_{T(N T)}(E, t)$ the $\Delta J_{T(N T)}(\omega, t)$ and subsequent $\Delta \epsilon_{I B}(\omega, t)$ (one from thermalized electrons and one from non-thermalized electrons) are retrieved as described in the main text.

Table 1: List of parameters used in the theoretical modeling of copper thermo-modulational nonlinearity. ${ }^{(*)}$ For this parameter we assumed the value reported for gold.

\begin{tabular}{|lr|r|r|r|}
\hline Parameter Name and Symbol & & Value & Unit & Refs. \\
\hline \hline Fermy Energy & $E_{F}$ & 9 & $\mathrm{eV}$ & 63 \\
Debye Temperature & $\theta_{D}$ & 343.5 & $\mathrm{~K}$ & 64 \\
Lattice Heat Capacity & $C_{L}$ & $3.44 \times 10^{6}$ & $\mathrm{~J} \mathrm{~m}^{-3} \mathrm{~K}^{-1}$ & 65 \\
Electron Heat Capacity Constant & $\gamma$ & 97 & $\mathrm{~J} \mathrm{~m}^{-3} \mathrm{~K}^{-2}$ & 64 \\
Quasi-Particle Free-Flight & $\tau_{f}$ & 12.3 & $\mathrm{fs}$ & 63,66 \\
Drude Damping Temperature Constant & $\beta$ & $3 \times 10^{-3}$ & $\mathrm{~K}^{-1}$ & 53 \\
Electron-Phonon Coupling Constant & $g$ & $1.3 \times 10^{17}$ & $\mathrm{~W} \mathrm{~m}^{-3} \mathrm{~K}^{-1}$ & 67,68 \\
Electron-Electron Scattering Time Constant & $\tau_{0}$ & 6 & $\mathrm{fs}$ & $34,69^{(*)}$ \\
\hline
\end{tabular}

\section{References}

(1) Lal, S.; Link, S.; Halas, N. J. Nano-optics from sensing to waveguiding. Nat. Photonics 2007, 1, 641-648.

(2) Schuller, J. A.; Barnard, E. S.; Cai, W.; Jun, Y.; White, J. S.; l. Brongersma, M. Plasmonics for extreme light concentration and manipulation. Nat. Mater. 2010, 9, 193-204.

(3) Gramotnev, D. K.; Bozhevolnyi, S. I. Plasmonics beyond the diffraction limit. Nat. Photonics 2010, 4, 83-91.

(4) Stewart, M. E.; Anderton, C. R.; Thompson, L. B.; Maria, J.; Gray, S. K.; Rogers, J. A.; Nuzzo, R. G. Nanostructured Plasmonic Sensors. Chem. Rev. 2008, 108, 494-521. 
(5) Anker, J. N.; Hall, W. P.; Lyandres, O.; Shah, N. C.; Zhao, J.; Van Duyne, R. P. Biosensing with plasmonic nanosensors. Nat. Mater. 2008, 7, 442-453.

(6) Atwater, H. A.; Polman, A. Plasmonics for improved photovoltaic devices. Nat. Mater. 2010, 9, 205-213.

(7) Awazu, K.; Fujimaki, M.; Rockstuhl, C.; Tominaga, J.; Murakami, H.; Ohki, Y.; Yoshida, N.; Watanabe, T. A plasmonic photocatalyst consisting of silver nanoparticles embedded in titanium dioxide. J. Am. Chem. Soc. 2008, 130, 1676-1680.

(8) Huang, X.; El-Sayed, I. H.; Qian, W.; El-Sayed, M. A. Cancer Cell Imaging and Photothermal Therapy in the Near-Infrared Region by Using Gold Nanorods. J. Am. Chem. Soc. 2006, 128, 2115-2120.

(9) Loo, C.; Lowery, A.; Halas, N.; West, J.; Drezek, R. Immunotargeted Nanoshells for Integrated Cancer Imaging and Therapy. Nano Lett. 2005, 5, 709-711.

(10) Sun, C.-K.; Vallée, F.; Acioli, L. H.; Ippen, E. P.; Fujimoto, J. G. Femtosecond-tunable measurement of electron thermalization in gold. Phys. Rev. B 1994-II, 50, 1533715348.

(11) Della Valle, G.; Conforti, M.; Longhi, S.; Cerullo, G.; Brida, D. Real-time optical mapping of the dynamics of nonthermal electrons in thin gold films. Phys. Rev. B 2012, 86, 155139 .

(12) Dal Conte, S.; Conforti, M.; Petti, D.; Albisetti, E.; Longhi, S.; Bertacco, R.; De Angelis, C.; Cerullo, G.; Della Valle, G. Disentangling electrons and lattice nonlinear optical response in metal-dielectric Bragg filters. Phys. Rev. B 2012, 89, 125122.

(13) Wurtz, G. A.; Pollard, R.; Hendren, W.; Wiederrecht, G. P.; Gosztola, D. J.; Podolskiy, V. A.; Zayats, A. V. Designed ultrafast optical nonlinearity in a plasmonic nanorod metamaterial enhanced by nonlocality. Nat. Nanotechnol. 2011, 6, 107-111. 
(14) Ren, M.; Jia, B.; Ou, J.-Y.; Plum, E.; Zhang, J.; MacDonald, K. F.; Nikolaenko, A. E.; Xu, J.; Gu, M.; Zheludev, N. I. Nanostructured Plasmonic Medium for Terahertz Bandwidth All-Optical Switching. Adv. Mater. 2011, 23, 5540-5544.

(15) MacDonald, K. F.; Samson, Z. L.; Stockman, M. I.; Zheludev, N. I. Ultrafast Active Plasmonics. Nat. Photonics 2009, 3, 55-58.

(16) Wang, X.; Morea, R.; Gonzalo, J.; Palpant, B. Coupling Localized Plasmonic and Photonic Modes Tailors and Boosts Ultrafast Light Modulation by Gold Nanoparticles. Nano Lett. 2015, 15, 2633-2639.

(17) Baida, H.; Mongin, D.; Christofilos, D.; Bachelier, G.; Crut, A.; Maioli, P.; Del Fatti, N.; Vallée, F. Ultrafast Nonlinear Optical Response of a Single Gold Nanorod near Its Surface Plasmon Resonance. Phys. Rev. Lett. 2012, 107, 057402-1-057402-5.

(18) Zavelani-Rossi, M.; Polli, D.; Kochtcheev, S.; Baudrion, A.-L.; Beál, J.; Kumar, V.; Molotokaite, E.; Marangoni, M.; Longhi, S.; Cerullo, G.; Adam, P.-M.; Della Valle, G. Transient Optical Response of a Single Gold Nanoantenna: The Role of Plasmon Detuning. ACS Photonics 2015, 2, 521-529.

(19) Boyd, R. W.; Shi, Z.; Leon, I. D. The third-order nonlinear optical susceptibility of gold. Opt. Comm. 2014, 326, 74-79.

(20) Kauranen, M.; Zayats, A. V. Nonlinear plasmonics. Nat. Photonics 2012, 6, 737-748.

(21) Naik, G. V.; Shalaev, V. M.; Boltasseva, A. Alternative Plasmonic Materials: Beyond Gold and Silver. Adv. Mater. 2013, 25, 3264-3294.

(22) Boltasseva, A.; Atwater, H. A. Ultrafast Active Plasmonics. Science 2011, 331, 290291.

(23) Comin, A.; Manna, L. New materials for tunable plasmonic colloidal nanocrystals. Chem. Soc. Rev. 2014, 43, 3957-3975. 
(24) Koppens, F. H.; Chang, D. E.; Garcia de Abajo, F. J. Graphene plasmonics: a platform for strong light-matter interactions. Nano Lett. 2011, 11, 3370-3377.

(25) Grigorenko, A. N.; Polini, M.; Novoselov, K. S. Graphene plasmonics. Nature Photonics 2012, 6, 749-758.

(26) Raman, R. S.; Banerjee, P. C.; Lobo, D. E.; Gullapalli, H.; Sumandasa, M.; Kumar, A.; Choudhary, L.; Tkacz, R.; Ajayan, P. M.; Majumder, M. Protecting copper from electrochemical degradation by graphene coating. Carbon 2012, 50, 4040-4045.

(27) Kravets, V. G.; Jalil, R.; Kim, Y.-J.; Ansell, D.; Aznakayeva, D. E.; Thackray, B.; Britnell, L.; Belle, B. D.; Withers, F.; Radko, I. P.; Han, Z.; Bozhevolnyi, S. I.; Novoselov, K. S.; Geim, A. K.; Grigorenko, A. N. Graphene-protected copper and silver plasmonics. Sci. Rep. 2014, 4, 5517.

(28) Weatherup, R. S.; D?Arsie, L.; Cabrero-Vilatela, A.; Caneva, S.; Blume, R.; Robertson, J.; Schloegl, R.; Hofmann, S. Long-Term Passivation of Strongly Interacting Metals with Single-Layer Graphene. J. Am. Chem Soc. 2015, 137, 14358-14366.

(29) Liu, X.; Wang, J.; Wu, Y.; Fan, T.; Xu, Y.; Tang, L.; Ying, Y. Compact Shielding of Graphene Monolayer Leads to Extraordinary SERS-Active Substrate with Large- Area Uniformity and Long-Term Stability. Sci. Rep. 2015, 5, 17167.

(30) Prasai, D.; Tuberquia, J. C.; Harl, R. R.; Jennings, G. K.; Bolotin, K. I. Graphene: corrosion-inhibiting coating. ACS Nano 2012, 6, 1102-1108.

(31) Sun, D.; Wu, Z.-K.; Divin, C.; Li, X.; Berger, C.; de Heer, W. A.; First, P. N.; Norris, T. B. Ultrafast Relaxation of Excited Dirac Fermions in Epitaxial Graphene Using Optical Differential Transmission Spectroscopy. Phys. Rev. Lett. 2008, 101, 157402.

(32) Breusing, M.; Kuehn, S.; Winzer, T.; Malic, E.; Milde, F.; Severin, N.; Rabe, J. P.; 
Ropers, C.; Knorr, A.; Elsaesser, T. Ultrafast nonequilibrium carrier dynamics in a single graphene layer. Phys. Rev. B 2011, 83, 153410.

(33) Shang, J.; Yu, T.; Lin, J.; Gurzadyan, G. G. Ultrafast Electron-Optical Phonon Scattering and Quasiparticle Lifetime in CVD-Grown Graphene. ACS Nano 2011, 5, 32783283.

(34) Conforti, M.; Della Valle, G. Derivation of third-order nonlinear susceptibility of thin metal films as a delayed optical response. Phys. Rev. B. 2012, 85, 245423-1-245423-4.

(35) Tajima, N.; Fukui, M.; Shintani, Y.; Tada, O. In situ studies on oxidation of copper films by using ATR technique. J. Phys. Soc. Japan 1985, 54, 4236-4240.

(36) Platzman, I.; Brener, R. R.; Haick, H.; Tannenbaum, R. Oxidation of Polycrystalline Copper Thin Films at Ambient Conditions. J. Phys. Chem. C 2008, 112, 1101-1108.

(37) Rhodin Jr., T. N. Low Temperature Oxidation of Copper. I. Physical Mechanism. J. Am. Chem. Soc. 1950, 72, 5102-5106.

(38) Shen, X.; Timalsina, Y. P.; Lu, T.-M.; Yamaguchi, M. Experimental study of electronphonon coupling and electron internal thermalization in epitaxially grown ultrathin copper films. Phys. Rev. B. 2015, 91, 045129.

(39) Anisimov, S. I.; Kapeliovich, B. L.; Perel'man, T. L. Electron emission from metal surfaces exposed to ultrashort laser pulses. Sov. Phys. JETP 1974, 39, 375-377.

(40) Cooper, B. R.; Ehrenreich, H. Optical Properties of Noble Metals. II. Phys. Rev. 1965, 138, A494-A507.

(41) Mueller, F. M.; Phillips, J. C. Electronic Structure of Crystalline Copper. Phys. Rev. 1967, 15\%, 600-607.

(42) Hartland, G. V. Optical Studies of Dynamics in Noble Metal Nanostructures. Chem. Rev. 2011, 111, 3858-3887. 
(43) Ehrenreich, H.; Philipp, H. R. Optical properties of Ag and Cu. Phys. Rev. 1962, 128, $1622-1629$.

(44) Motulevich, G. P. Optical properties of metals, d. v. skobel'tsyn ed.; Consultants Bureau: New York, 1973.

(45) Ordal, M. A.; Bell, R. J.; Alexander Jr, R. W.; Long, L. L.; Querry, M. R. Optical properties of fourteen metals in the infrared and far infrared: $\mathrm{Al}, \mathrm{Co}, \mathrm{Cu}, \mathrm{Au}, \mathrm{Fe}, \mathrm{Pb}$, Mo, Ni, Pd, Pt, Ag, Ti, V, and W. Appl. Opt. 1985, 24, 4493-4499.

(46) Zeman, E. J.; Schatz, G. C. An Accurate Electromagnetic Theory Study of Surface Enhancement Factors for Ag, Au, Cu, Li, Na, Al, Ga, In, Zn, and Cd. J. Phys. Chem. 1987, 91, 634-643.

(47) Chen, K.-P.; Drachev, V. P.; Borneman, J. D.; Kildishev, A. V.; Shalaev, V. M. Drude Relaxation Rate in Grained Gold Nanoantennas. Nano Lett. 2010, 10, 916-922.

(48) Kuryoz, P. Y.; Poperenko, L. V.; Kravets, V. G. Correlation between dielectric constants and enhancement of surface plasmon resonances for thin gold films. Phys. Status Solidi A 2013, 210, 2445-2455.

(49) Guerrisi, M.; Rosei, R.; Winsemius, P. Splitting of the interband absorption edge in Au. Phys. Rev. B 1975, 12, 557-563.

(50) Marini, A.; Conforti, M.; Della Valle, G.; Lee, H.; Tran, T. X.; Chang, W.; Schmidt, M. A.; Longhi, S.; Russell, P. S. J.; Biancalana, F. Ultrafast nonlinear dynamics of surface plasmon polaritons in gold nanowires due to the intrinsic nonlinearity of metals. New. J. Phys. 2013, 15, 013033-1-013033-19.

(51) Eckardt, H.; Fritsche, L.; Noffke, J. Self-consistent relativistic band structure of noble metals. J. Phys. F.: Met. Phys. 1984, 14, 97-112. 
(52) Yeh, P. Optical Waves in Layered Media, 1st ed.; Wiley: New York, 1988; Chapter V, pp 102-112.

(53) Beach, R. T.; Christy, R. W. Electron-electron scattering in the intraband optical conductivity of $\mathrm{Cu}, \mathrm{Ag}$, and Au. Phys. Rev. B 1977, 16, 5277-5284.

(54) Rosei, R. Temperature modulation of the optical transitions involving the Fermi surface in Ag: Theory. Phys. Rev. B 1974, 10, 474-483.

(55) Brida, D.; Tomadin, A.; Manzoni, C.; Kim, Y. J.; Lombardo, A.; Milana, S.; Nair, R. R.; Novoselov, K.; Ferrari, A. C.; Cerullo, G.; Polini, M. Ultrafast collinear scattering and carrier multiplication in graphene. Nature Communications 2013, 4, 1987-1-9.

(56) Breusing, M.; Ropers, C.; Elsaesser, T. Ultrafast Carrier Dynamics in Graphite. Phys. Rev. Lett. 2009, 102, 086809.

(57) Gao, C.; Zhao, X.; Yao, J.; Yan, X.-Q.; Kong, X.-T.; Chen, Y.; Liu, Z.-B.; Tian, J.-G. Sign of differential reflection and transmission in pump-probe spectroscopy of graphene on dielectric substrate. Photon. Res. 2015, 3, A1-A9.

(58) Leon, I. D.; Sipe, J. E.; Boyd, R. W. Self-phase-modulation of surface plasmon polaritons. Phys. Rev. A 2014, 89, 013855.

(59) Lysenko, O.; Bache, M.; Lavrinenko, A. Third-order susceptibility of gold for ultrathin layers. Opt. Lett. 2016, 41, 317-320.

(60) Li, X.; Cai, W.; An, J.; Kim, S.; Nah, J.; Yang, D.; Piner, R.; Velamakanni, A.; Jung, I.; Tutuc, E.; Banerjee, S. K.; Colombo, L.; Ruoff, R. S. Large-Area Synthesis of HighQuality and Uniform Graphene Films on Copper Foils. Science 2009, 324, 1312-1314.

(61) Polli, D.; Luer, L.; Cerullo, G. High-time-resolution pump-probe system with broadband detection for the study of time-domain vibrational dynamics. Rev. Sci. Instrum. 2007, 78, 103108. 
(62) Carpene, E. Ultrafast laser irradiation of metals: Beyond the two-temperature model. Phys. Rev. B 2006, 74, 024301.

(63) Halas, S.; Durakiewicz, T. Work functions of elements expressed in terms of the Fermi energy and the density of free electrons. J. Phys.: Condens. Matter 1998, 10, 1081510826.

(64) Billings, B. H.; Gray., D. E. American Institute of Physics Handbook, 3rd ed.; McGrawHill: New York, 1972.

(65) Lide, D. R. CRC Handbook of Chemistry and Physics, 85th ed.; CRC Press, 2004.

(66) Ashcroft, N. W.; Mermin, N. D. Solid State Physics, 1st ed.; Harcourt College Publisher: Forth Worth, 1976; Chapter I, pp 8-9.

(67) Eesley, G. L. Generation of nonequilibrium electron and lattice temperatures in copper by picosecond laser pulses. Phys. Rev. B 1986, 33, 2144-2151.

(68) Lin, Z.; Zhigilei, L. V.; Celli, V. Electron-phonon coupling and electron heat capacity of metals under conditions of strong electron-phonon nonequilibrium. Phys. Rev. B 2008, 7ry, 075133.

(69) Fann, W. S.; Storz, R.; Tom, H. W. K.; Bokor, J. Electron thermalization in gold. Phys. Rev. B 1992, 46, 13592-13595. 


\section{Graphical TOC Entry}

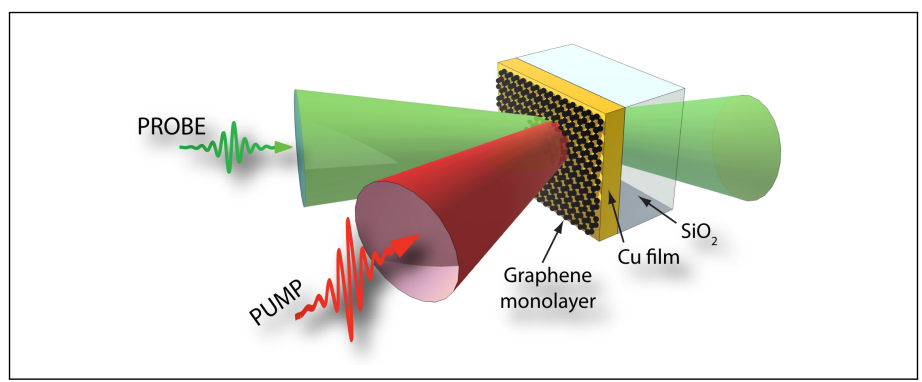

